and parkinsonism, akathisia and tardive dyskinesia were diagnosed using predefined scores. Twelve of the 18 patients (67\%) prescribed an atypical antipsychotic and 6 of the 7 patients (86\%) prescribed a conventional antipsychotic fulfilled criteria for an extrapyramidal syndrome. A subsequent review of the clinical notes for the preceding 12 months showed that only half of the patients with an extrapyramidal syndrome (9 of 18) had documented evidence of a management plan to treat the disorder and that only 1 of the 25 patients had a documented physical examination that recorded the presence or absence of extrapyramidal signs.

Our study indicates that EPS remain common in clinical practice despite the widespread use of atypical agents, that half of extrapyramidal syndromes are untreated and that screening for EPS is not routine. Possible explanations are that clinicians lack the knowledge, skills and confidence to assess and manage EPS or that they lack the time to address this aspect of care. We agree with Kuruvilla et al that clinicians need better education and training in this area.

Sabyasachi Mitra Consultant Psychiatrist, The Calcutta Medical Research Institute, West Bengal, India, *Peter Haddad Consultant Psychiatrist, Bolton, Salford and Trafford Mental Health NHS Trust, Salford, email: peterhaddad@doctors.org.uk

doi: 10.1192/pb.bp.31.2.77

\section{Paperless working environment - a logistical nightmare?}

I belong to a generation of psychiatrists who have learned to love the computer. We are also apparently proficient at using it and would naturally welcome the era of the electronic patient record.
Over the past few months, some trusts have introduced the RiO system, which creates an integrated electronic care record of patients across London (NPfIT; http://www.connectingforhealth.nhs.uk) The positive impact on our working lives is undeniable once the system is fully up and running: $24 \mathrm{~h}$ access to essential information across a wide geographical area would facilitate risk assessments and emergency care planning. We will also rid ourselves of the piles of indecipherable files cluttering our wards.

On closer inspection the trend carries practical implications that are yet to be addressed since investment in computers and related equipment has not followed. Few junior doctors have access to dedicated offices and often six to seven senior house officers share one terminal and an antiquated printer. These juniors, who already struggle to balance clinical duties and academic requirements, will have to find the time and the facilities to input information on a daily basis.

Ideally all doctors should be issued with laptops or hand-held devices. Alternatively administrative personnel could provide instant support to clinical staff. None of these scenarios is likely in the near future in a cash-strapped National Health Service. None the less imaginative solutions are required if we are to avoid the dream of a paperless work environment turning into a logistical nightmare.

Joseph El-Khoury Senior House Officer in Psychiatry, St George'sTraining Scheme, London, email: Joseph.elkhoury@wlmht.nhs.uk

doi: 10.1192/pb.bp.31.2.77a

\section{Sanctions on clients who use opiates on top of methadone}

I agree with Dunn et al (Psychiatric Bulletin, September 2006, 30, 337-339) that statutory drug services cannot meet all the needs of homeless people, who have chaotic lifestyles and multiple physical health, mental health and social needs. In view of this the outreach clinic model offers an innovative way of engaging this group of clients.

We know that methadone maintenance has a strong evidence base in harm reduction (Luty, 2003). The outcomes described by Dunn et al probably could be attributed to this, as evidenced by the decreased use of heroin, the amount of money spent and the number of injections per day.

However, I was not convinced that sanctions should not be imposed for continued opiate use, given the risk of overdosing. I appreciate that a harm reduction model is more concerned with reducing harm than achieving abstinence. The range of heroin use among the clients was quite wide (amount spent per day $\mathrm{f} 10-\mathrm{f} 200$ ). It is not clear whether the drop in heroin use and number of injections was a direct result of clients who stopped using opiates altogether. I would be interested to know whether any harm reduction actually took place in people who continued to use opiates on top of methadone.

Although I agree that there should be some leniency in the imposition of sanctions with this difficult group of clients, I feel that sanctions should be used at some point, if the methadone prescription is not achieving anything.

UTY, J. (2003) What works in drug addiction? Advances in Psychiatric Treatment, 9, 280-288.

Sandeep Shastry Staff Grade Psychiatrist, Leeds Addiction Unit, Leeds, email: sandeep830@ yahoo.com

doi: 10.1192/pb.bp.31.2.77b 